\title{
SpinQuest/E1039 FPGA Trigger
}

Minjung Kim

University of Michigan

On behalf of SpinQuest collaboration
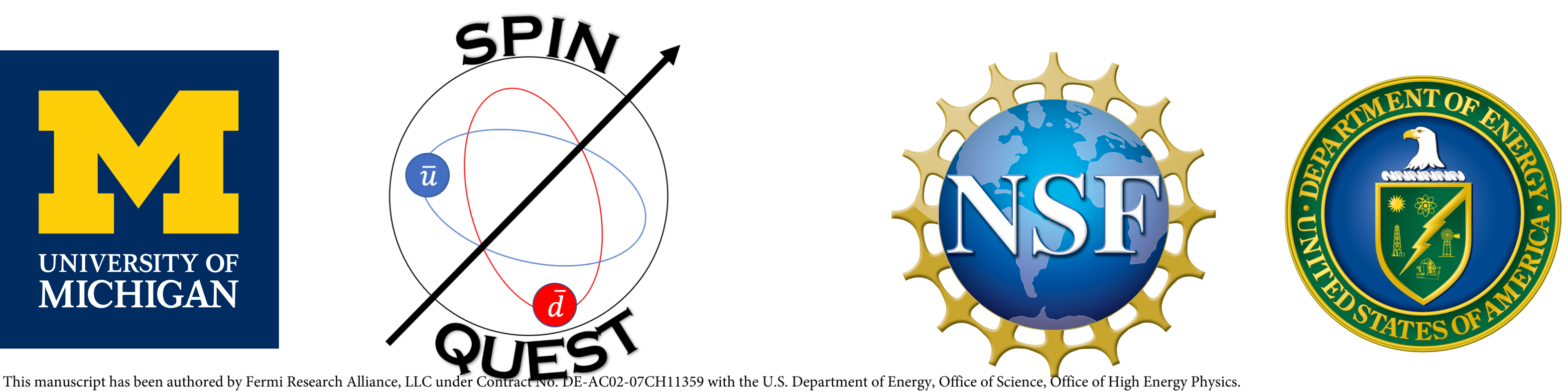


\section{SpinQuest/E1039 experiment}

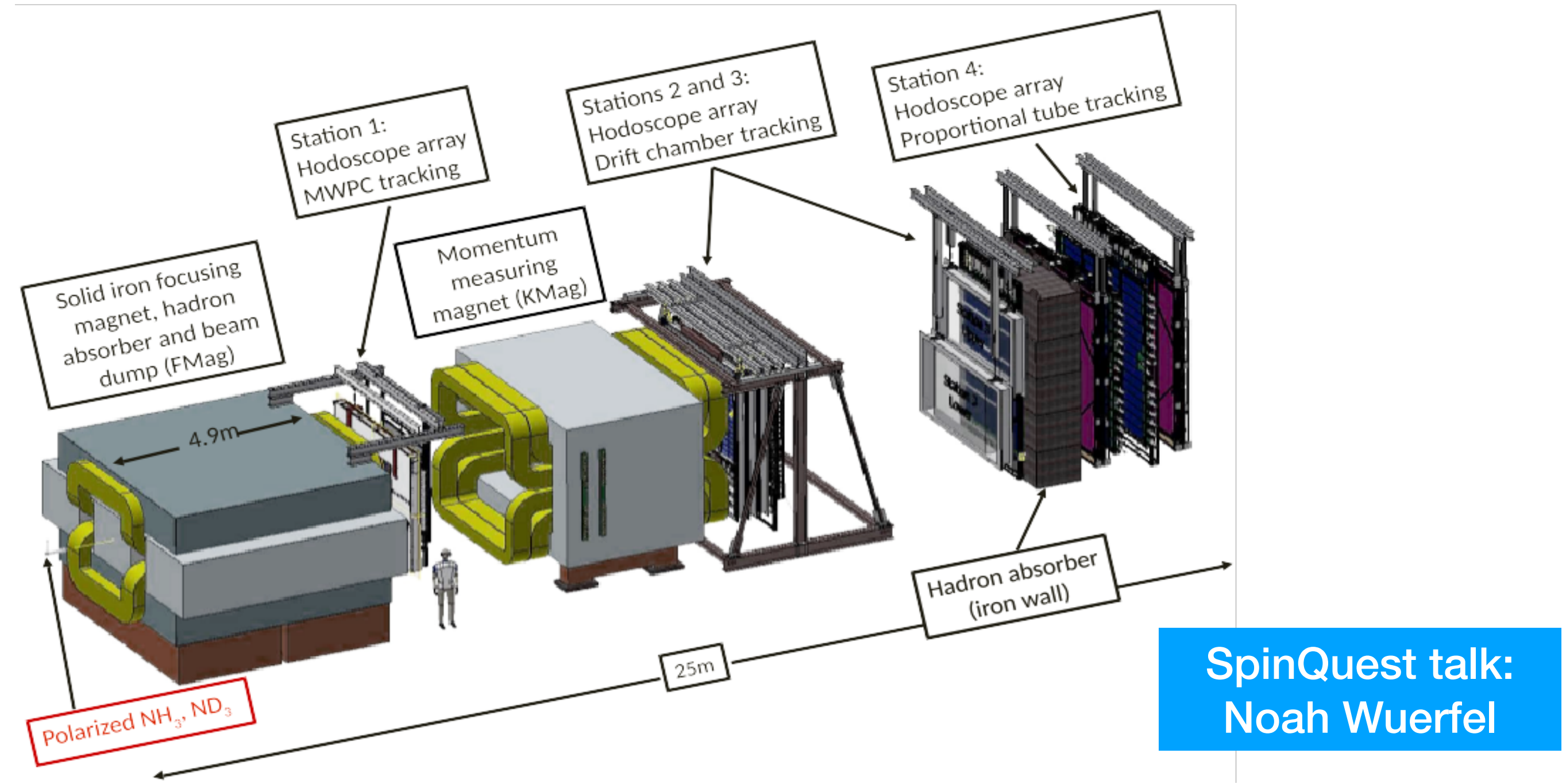

- To extract Sivers function of ubar \& dbar in a nucleon through DY decayed dimuon asymmetry measurement

- Dimuon detection by 4 stations of tracker \& hodoscope scintillators and hadron absorber

- Requires a trigger to select DY dimuon under high fraction \& rate of background in total muon flux (due to interaction with beam dump, rareness of DY process): designed to select oppositelycharged muon pairs at 4-9 GeV mass range 


\section{FPGA trigger principle}

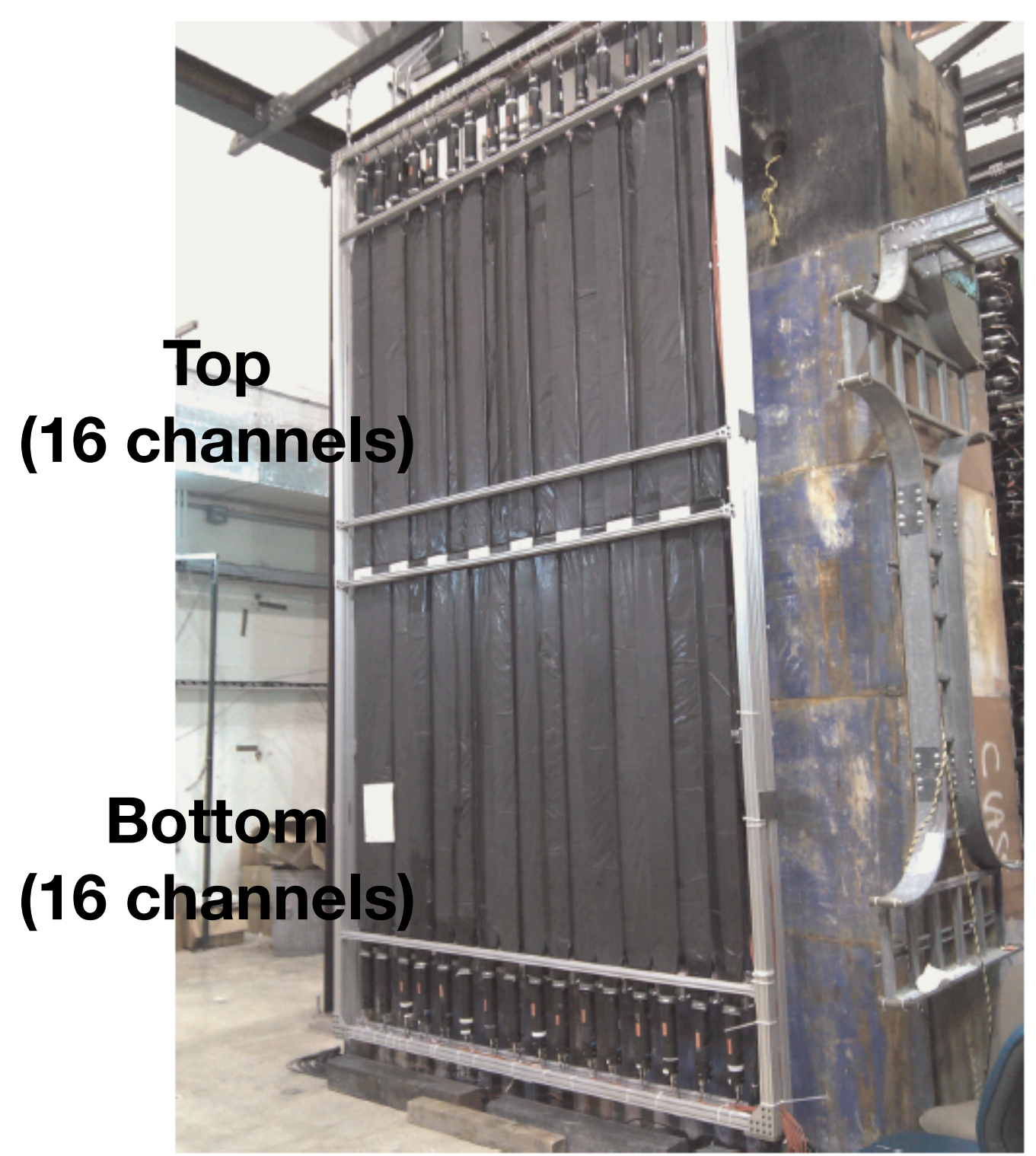

Scintillation hodoscope arrays for X (horizontal) - position at station 3
- Consist of scintillator hodoscope + FPGA based VMEbus modules

- Get X-position of muon from arrays of vertical scintillators

- Compare hit patterns of 4 stations of hodoscopes with predefined hit patterns (DY dimuon "roads") obtained from Monte Carlo simulation to generate trigger decisions

- These roads form a tiered Look-up-table in the FPGA

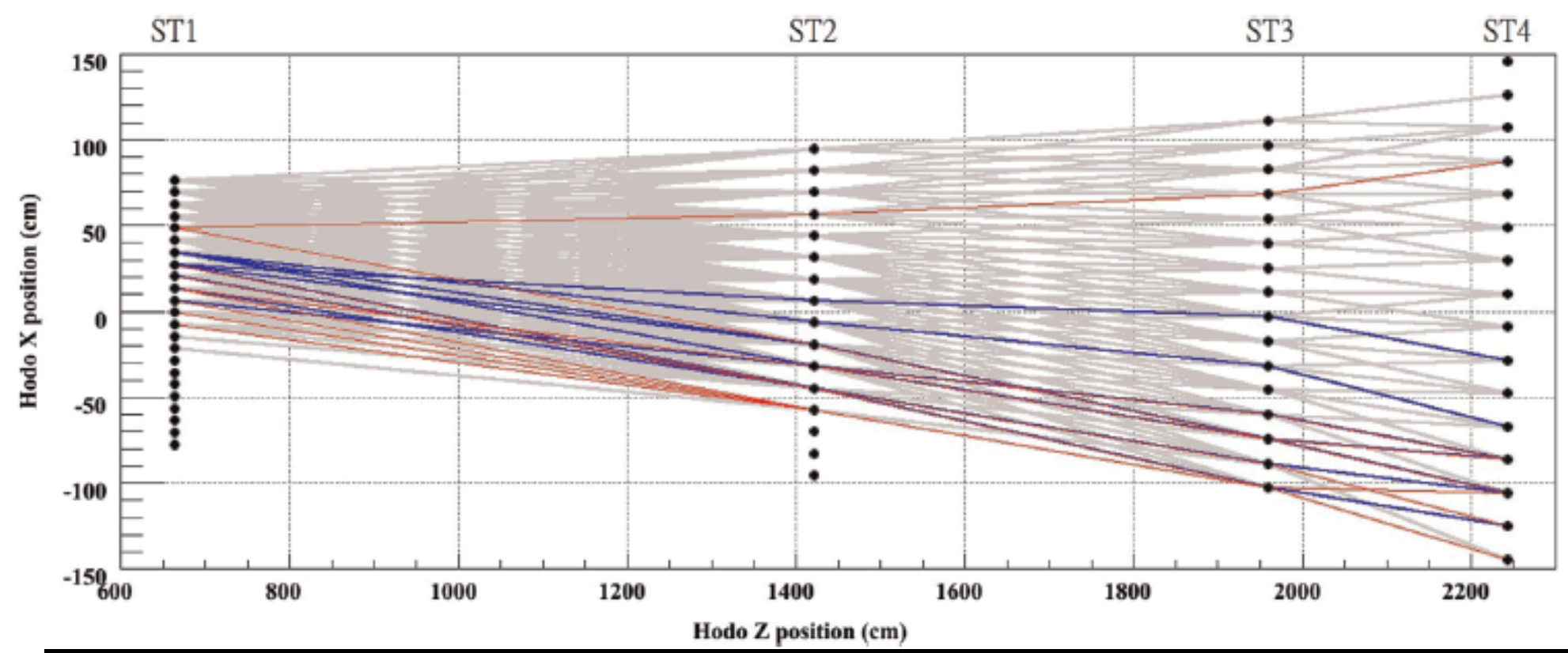

Visualized hit patterns (roads) of positive muons from E906/ SeaQuest MC simulation study

Black dots: scintillator paddles viewed from the top Red (blue) lines: the 10 most (the next 10 most) frequent roads 


\section{Trigger board - CAEN V1495}

- VMEbus module, contains user programmable FPGA with 20,060 logic elements $(<1000$ pre-defined roads per board)

- Takes up to 96 channels of inputs (16 channels $x 4$ stations

$=$ total 72 channels of hodoscope input for each top/ bottom board)

- Total 5 boards consists FPGA trigger system

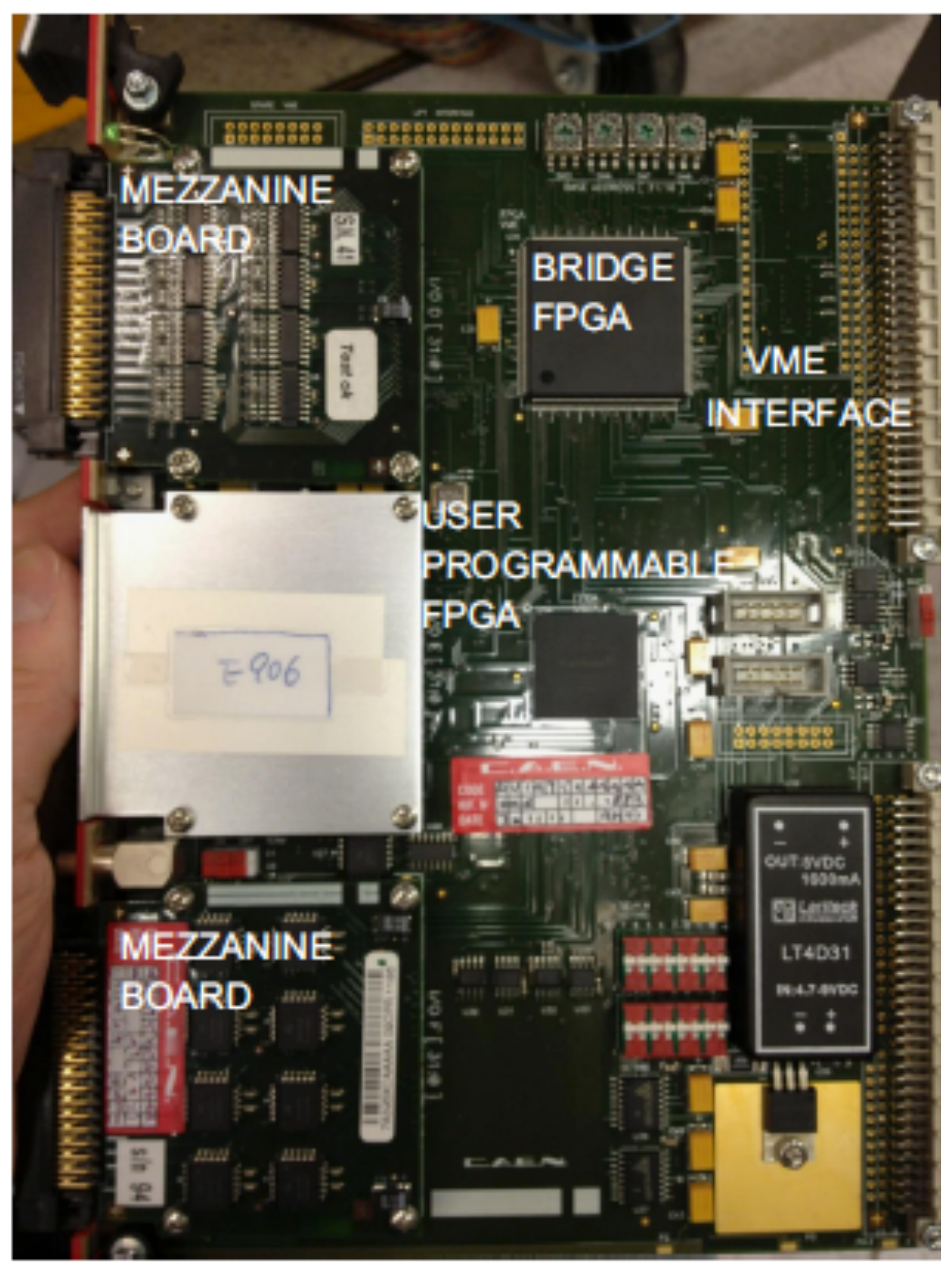




\section{FPGA trigger block diagram}

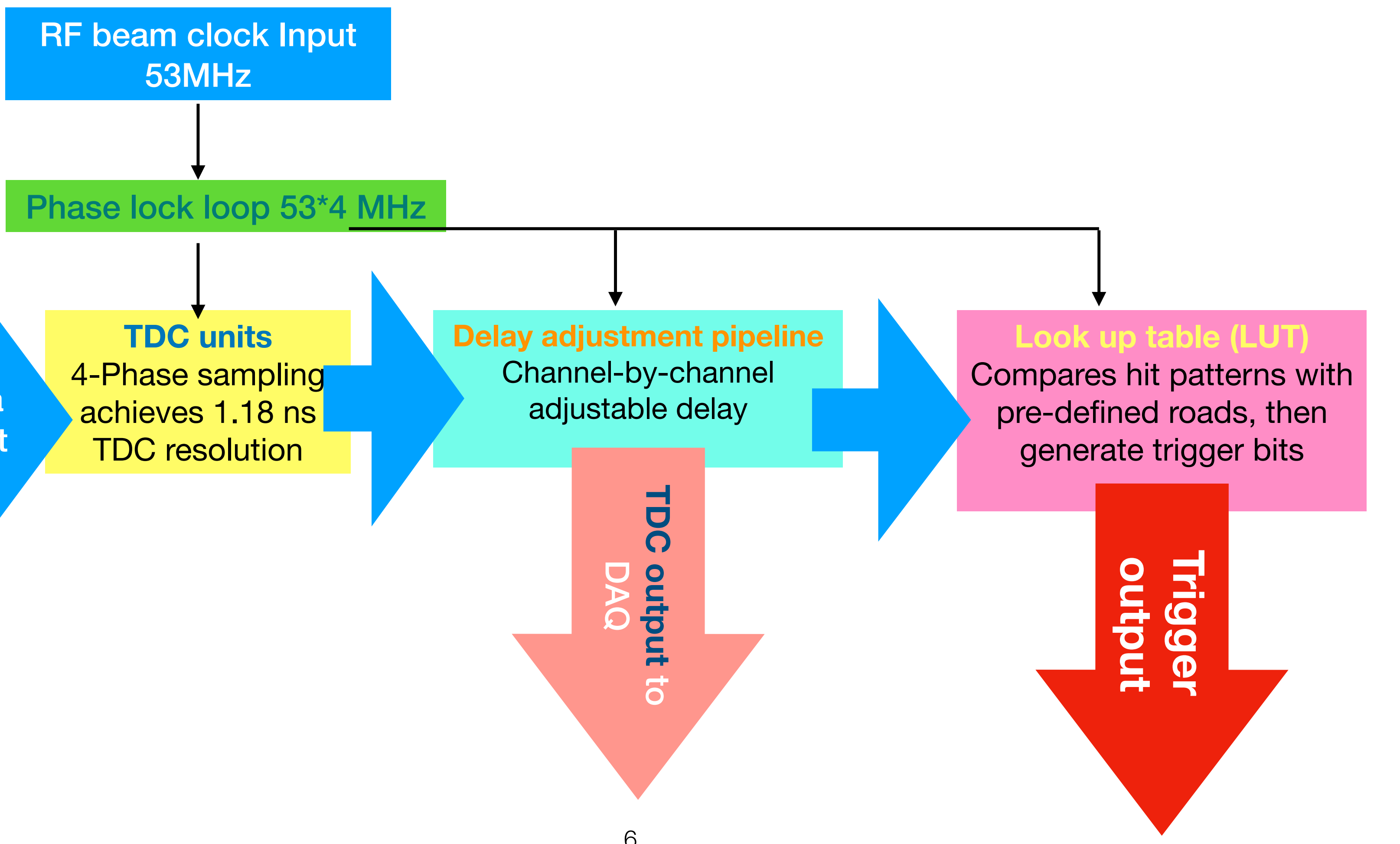




\section{SpinQuest trigger status}

- Took over decommissioned e906 trigger in 2019

- Tested trigger functionality at test bench setup

- Integrated to DAQ and tested with cosmic data

- TDC pipeline delay adjusted with cosmic muon \& pulser generator

- Simulation study is ongoing to optimize Look Up Table 


\section{TDC delay adjustment}

Hit timing (TDC unit)

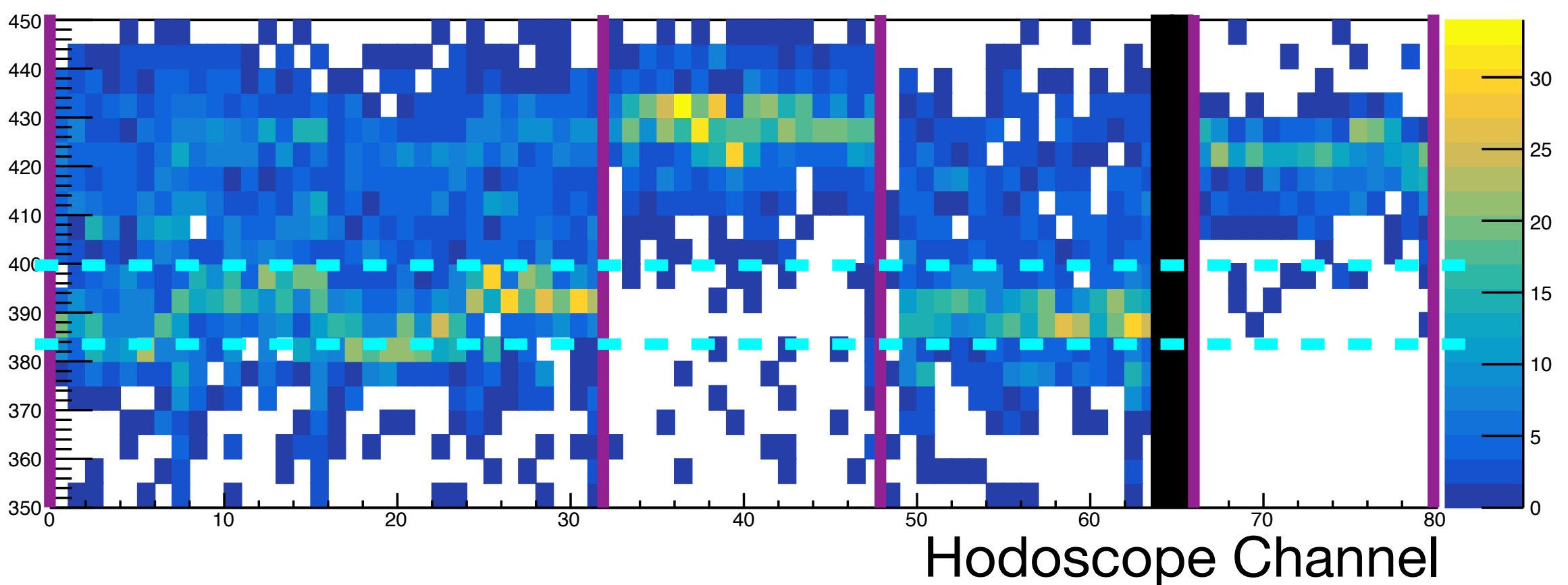

After timing tuned

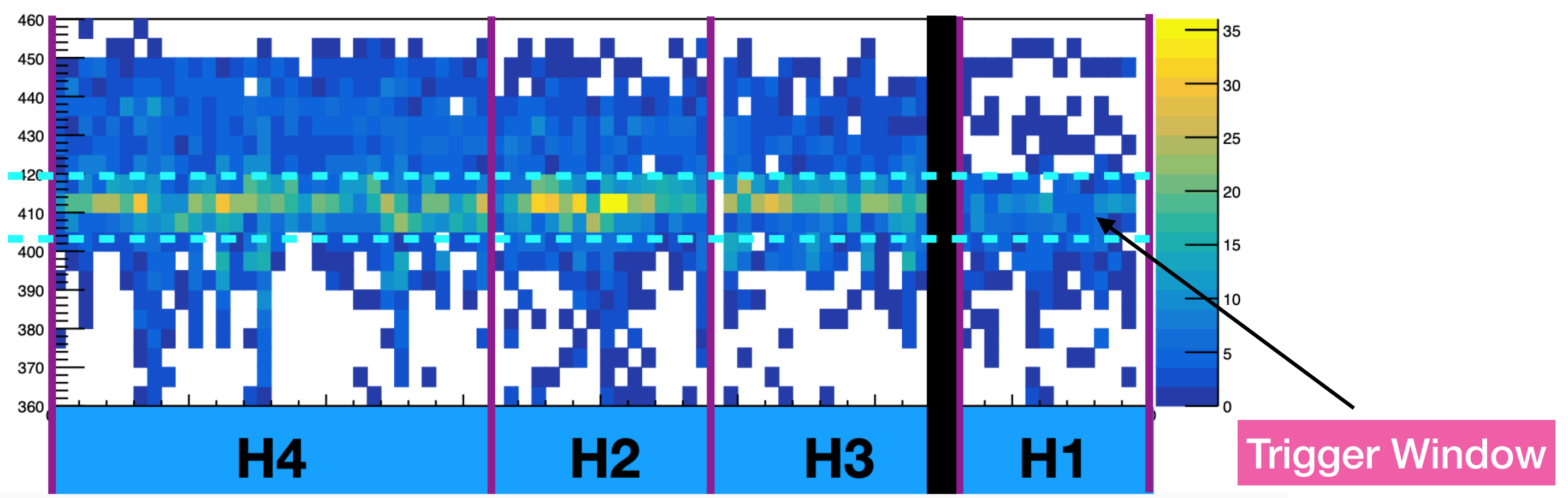




\section{Look up table study}

- Background in E906 setup: scattering at beam dump, combinatorial muons decayed from pions

- E906 setup didn't have enough vertex resolution to separate target and beam dump. Therefore, E1039 target was moved $\sim 3$ m upstream.

- Pions have low $p_{T}$, seem to come from soft processes. Adding yhodoscope information is expected to reduce such coincidence background.

- To improve trigger performance by adding y-hodoscope information to LUT, simulation study is ongoing to estimate efficiency and purity. Expected to be done by the end of 2020 . 


\section{Summary}

- SpinQuest FPGA trigger is designed to select DY dimuon events at high background rate.

- The trigger decision is made based on hodoscope hit pattern compared with pre-defined hit patterns obtained from MC simulation (dimuon roads).

- Simulation study is ongoing to suppress high combinatorial background events efficiently.

- FPGA trigger expected to be ready for production data by end of 2020. 


\section{Thank you}




\section{Back up}




\section{FPGA trigger block diagram}

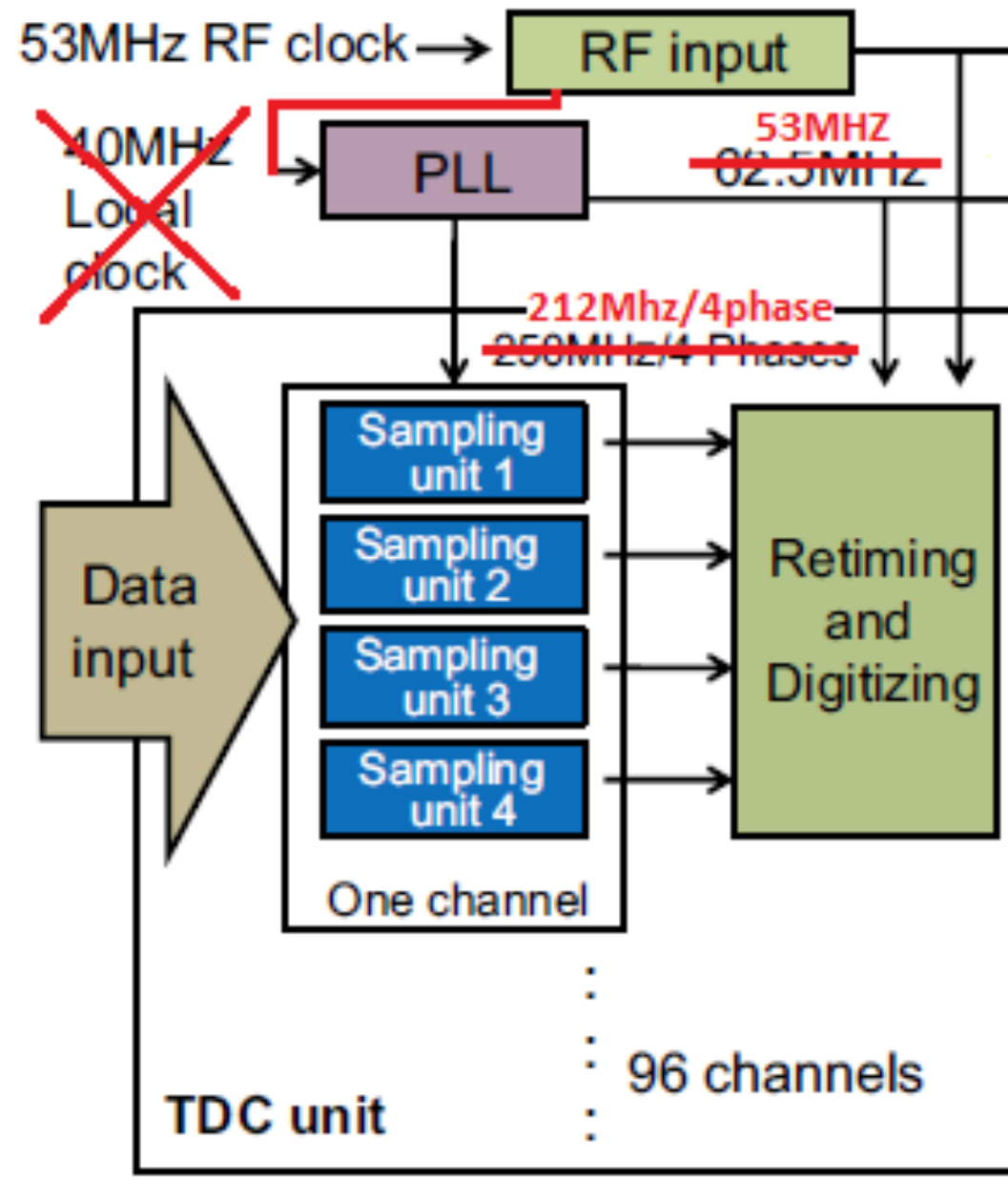

TDC

Clocked by RF, Phase Lock Loop \& 4-phase sampling achieves 1.18 ns TDC resolution

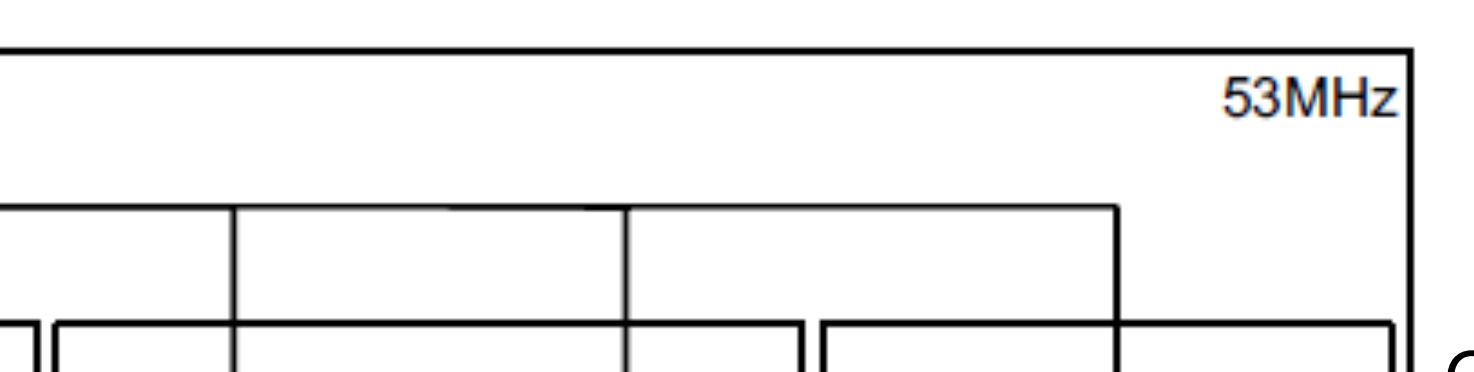

Look up table (LUT) Compares hit patterns with pre-defined roads, then generate trigger bits

\section{Lvl B}

"AND" operation for each road (ch st1 \& ch st2 \& ch st3 $\underline{\&}$ ch st4) Then "OR" operation for all roads (road1 or road2 or ...)

Lvl C [Top mu+ \& Bottom mu-] or

Old design crossed out
Delay adjustment pipeline Channel-by-channel adjustable delay TDC output written to DAQ machine decoded to online monitoring

[Top mu- \& Bottom mu+]

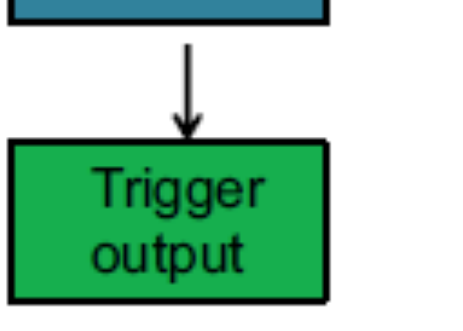




\section{Pulser generator setup}

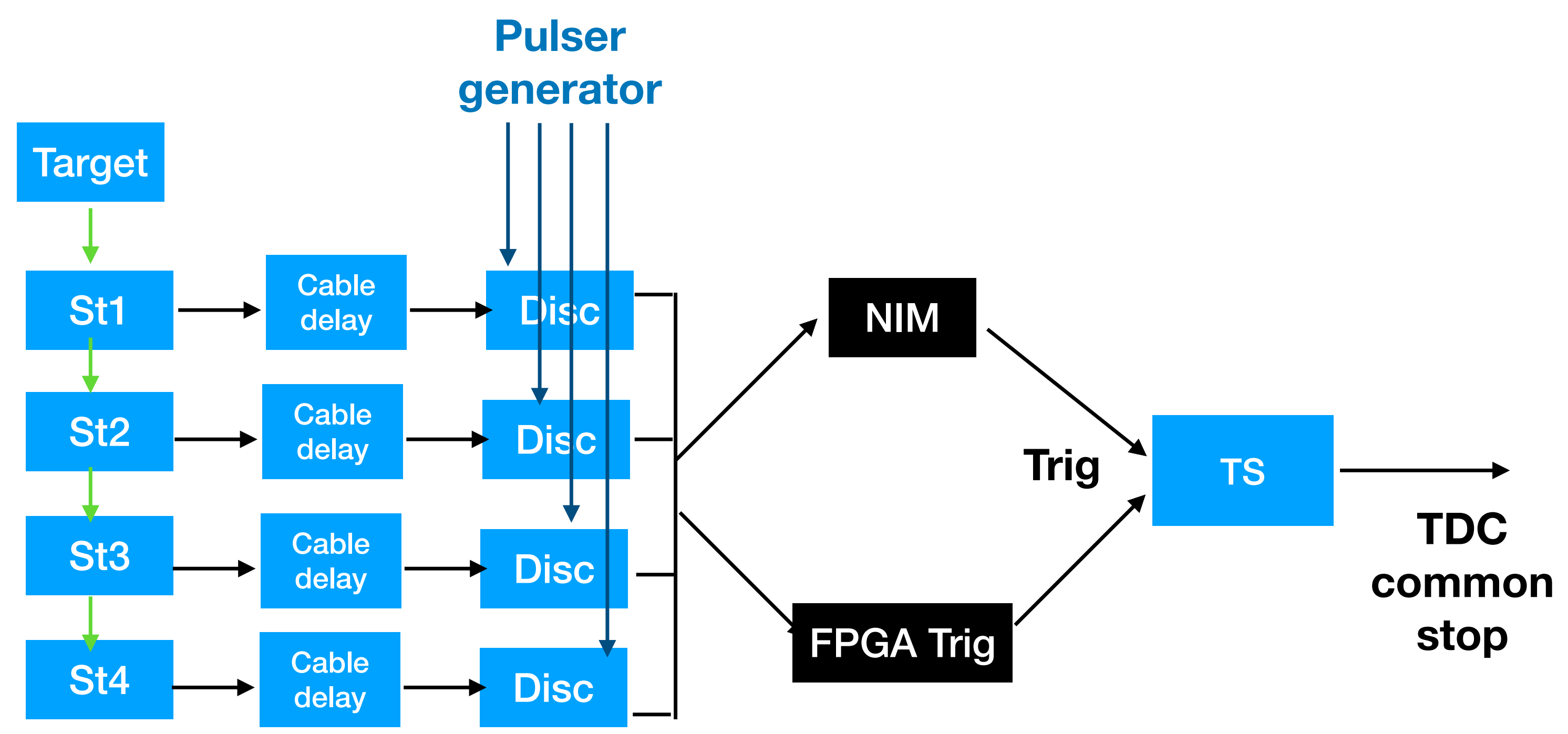

Difficult to tune timing with cosmic ray due to high background that hits partial hodoscope stations Emulate real muon signal timing using pulser generator 